HIV Clinical Trials

\title{
Reduced Plasma Levels of SCD14 and I-FABP in HIV-infected Patients with Mesalazine-treated Ulcerative Colitis
}

Zuleika Michelini, Silvia Baroncelli, Alessandra Fantauzzi, Chiara Pasquale, Clementina Maria Galluzzo, Massimo Sanchez, Manuela Gatto, Roberta Amici, Marina Franco, Gabriella d'Ettorre, Caterina Fimiani, Ivano Mezzaroma, Vincenzo Vullo, Manuela Merli \& Lucia Palmisano

To cite this article: Zuleika Michelini, Silvia Baroncelli, Alessandra Fantauzzi, Chiara Pasquale, Clementina Maria Galluzzo, Massimo Sanchez, Manuela Gatto, Roberta Amici, Marina Franco, Gabriella d'Ettorre, Caterina Fimiani, Ivano Mezzaroma, Vincenzo Vullo, Manuela Merli \& Lucia Palmisano (2016) Reduced Plasma Levels of SCD14 and I-FABP in HIV-infected Patients with Mesalazine-treated Ulcerative Colitis, HIV Clinical Trials, 17:2, 49-54, DOI: 10.1080/15284336.2015.1125077

To link to this article: http://dx.doi.org/10.1080/15284336.2015.1125077

册 Published online: 07 Jan 2016.

Submit your article to this journal $\pi$

Џll Article views: 49

Q View related articles $\sqsubset$

View Crossmark data ¿ 


\title{
Reduced Plasma Levels of sCD14 and I-FABP in HIV-infected Patients with Mesalazine- treated Ulcerative Colitis
}

\section{Zuleika Michelini ${ }^{1 \star}$, Silvia Baroncelli ${ }^{1 *}$, Alessandra Fantauzzi², Chiara Pasquale ${ }^{3}$, Clementina Maria Galluzzo, Massimo Sanchez ${ }^{4}$, Manuela Gatto ${ }^{3}$, Roberta Amici ${ }^{1}$, Marina Franco ${ }^{1}$, Gabriella d'Ettorre', Caterina Fimiani', Ivano Mezzaroma ${ }^{3}$, Vincenzo Vullo 6 , Manuela Merli³, Lucia Palmisano ${ }^{1}$}

\begin{abstract}
${ }^{1}$ Department of Therapeutic Research and Medicines Evaluation, Istituto Superiore di Sanità, Rome, Italy, ${ }^{2}$ Department of Clinical Medicine, Sapienza University of Rome, Rome, Italy, ${ }^{3}$ Gastroenterology, Department of Clinical Medicine, Sapienza University of Rome, Rome, Italy, ${ }^{4}$ Department of Cell Biology and Neurosciences, Istituto Superiore di Sanità, Rome, Italy, ${ }^{5}$ Department of Infectious and Tropical Diseases, Policlinico Umberto I, Rome, Italy, ${ }^{6}$ Department of Public Health and Infectious Diseases, Sapienza University of Rome, Rome, Italy
\end{abstract}

Background: Microbial translocation (MT) is a shared feature of HIV infection and inflammatory bowel disease (IBD). Aims: This study was conducted to assess the impact of IBD (and particularly ulcerative colitis, UC) on plasma markers of MT and immune activation in HIV+ subjects.

Methods: A cross-sectional study was conducted in 3 groups of patients: HIV+/UC+(group HIV/UC); HIV+/UC(group HIV); HIV-/UC+(group UC). Plasma levels of soluble CD14 (sCD14), intestinal fatty acid-binding protein (I-FABP), and endotoxin core antibodies (endoCAB) were measured as plasma markers of MT. Inflammation and immune activation were evaluated by measuring plasma levels of IL-6, IL-21, TNF-alpha, and high-sensitivity C-reactive protein (hs-CRP). T- and B-cells subpopulations were characterized by FACS analysis.

Results: Seven patients were enrolled in group HIV/UC, 9 in HIV, and 10 in UC. All HIV-positive patients had plasma values of HIV-1 RNA < 37 copies $/ \mathrm{mL}$ for at least 12 months and good immunological recovery. All patients with UC were treated with oral mesalazine. Markers of MT, immune activation, and inflammation were not increased in subjects with HIV/UC. In fact, they had lower levels of I-FABP $(p=0.001)$ and SCD14 ( $p=0.007)$ when compared to other patients groups. Positive correlations were found between I-FABP and SCD14 $(r=.355$, $p=0.076)$. Frequency of T- and B-cell subsets did not differ among groups.

Conclusions: Our results suggest that UC does not worsen MT, inflammation, or immune activation in HIVinfected subjects. The anti-inflammatory activity of chronic mesalazine administration on intestinal mucosa may contribute to this finding.

Keywords: Microbial translocation, HIV infection, Inflammatory bowel disease, Ulcerative colitis, Soluble CD14

\section{Introduction}

Gut associated lymphoid tissue (GALT) is one of the major early targets of HIV infection and an important site of HIV persistence during successful antiretroviral therapy (ART) ${ }^{1,2}$ From a clinical point of view patients may be asymptomatic or suffer from the so-called HIV enteropathy, which remains an important cause of acute and chronic non infectious diarrhea, affecting an unknown albeit significant proportion of HIV-infected individuals. ${ }^{3}$

Loss of gastrointestinal (GI) mucosal integrity leads to microbial translocation (MT) which results in the

Correspondence to: Lucia Palmisano, Department of Therapeutic Research and Medicines Evaluation, Istituto Superiore di Sanità, Rome, Viale Regina Elena 299, Rome, Italy. Email: lucia.palmisano@iss.it ${ }^{*}$ Contributed equally to this work. migration of luminal antigens, including microbial products, into the peripheral circulation. As a consequence, microbial antigens can directly stimulate the immune system and trigger a cascade of events leading to systemic immune activation. ${ }^{4}$ MT is not unique to HIV disease, being a well note feature in other conditions, such as chronic liver diseases ${ }^{5-8}$ and inflammatory bowel disease (IBD). ${ }^{9}$ This latter is characterized by a chronic inflammatory status of intestinal mucosa and can present itself in two different forms: Crohn's disease (CD) and ulcerative colitis (UC).

Different mechanisms contribute to MT in IBD and in HIV infection. In IBD, the disruption of the GI epithelium is associated with an autoimmune process triggered 
by unknown causes in a genetically predisposed individual; ${ }^{10}$ in HIV infection, the direct effect of HIV on enterocytes and the local activation of GALT are involved. Irrespective of the cause, the two conditions share some characteristics, including increased levels of markers of immune activation, inflammation, and MT. In addition, a IBD-like serological pattern was reported in $65 \%$ of $\mathrm{HIV}+$ patients with high blood levels of lipopolysaccharide (LPS). ${ }^{11}$ These observations have induced some authors to suggest HIV testing in subjects with IBD. ${ }^{12}$

Scarce data exist on the prevalence of IBD in HIV+ population, although in a large cohort of seropositive individuals in UK, the prevalence of UC was double than in uninfected population, ${ }^{13}$ and few studies have investigated the interplay between IBD and HIV infection in subjects fully responding to ART. Studies in small groups or individual patients with both HIV infection and IBD in the early years of ART era reported no worsening of immunological conditions, and an apparently more favorable course of IBD has been described. ${ }^{14-17}$ A more recent study confirmed the previous findings, describing a reduction in relapse rates in immunocompromised subjects with IBD and HIV..$^{18}$

Here, we report the results of a cross-sectional study where we assessed the impact of IBD in HIV+ subjects by measuring markers of MT and inflammation in plasma and levels of immune activation both in plasma and in circulating CD4+ and CD8+ T cells. In addition, considering the presence of B-cell dysfunction both in HIV infection and in IBD, B-cell phenotype was analyzed in all patients.

\section{Methods \\ Subjects}

Case records of $1300 \mathrm{HIV}+$ subjects attending the outpatient clinic at the Department of Public Health and Infectious Diseases (Sapienza University of Rome) were reviewed to identify those with concomitant IBD; 9 patients, all with histologically proven UC, were found $(9 / 1300,0.69 \%)$ and were asked to participate in this cross-sectional study; 2 of them refused to participate (group HIV/UC, $n=7$ ). Nine patients with HIV infection, matched for HIV history and demographic characteristics, were enrolled as controls (group HIV). A third group (group UC) of subjects included 10 uninfected individuals with UC, recruited from the Department of Clinical Medicine (Sapienza University of Rome). The study was approved by Ethics Committee of, Sapienza University Policlinico Umberto I ${ }^{\circ}$ Rome (Ref. 2789, prot. 915/13). All subjects gave their written informed consent

\section{Blood sampling and processing}

Twenty $\mathrm{ml}$ of peripheral blood was drawn from patients. EDTA-blood was processed on the same day of phlebotomy. Peripheral blood mononuclear cells (PBMC) were obtained by Ficoll gradient centrifugation (Lympholyte, Cederlane Laboratories, Ontario, Canada) and analyzed the same day. Plasma was collected and stored at $-80^{\circ}$ until required for analysis.

\section{MT and enterocytes damage biomarkers}

Soluble sCD14 (sCD14, R\&D Systems, Minneapolis, MN, USA), EndoCAb IgG (EndoCab Human, ELISA Hycult biotech, Uden, the Netherlands), and Intestinal fatty acid-binding protein (I-FABP, Hycult biotech, Uden, the Netherlands) were measured in plasma according to the manufacturer's instructions.

\section{Inflammation and immune activation markers}

Commercially available enzyme-linked immunoassorbent assay (ELISA) was used for measuring plasma amount of high-sensitive C-reactive protein (hs-CRP, eBioscience, Vienna Austria), IL-6 (R\&D Systems, Minneapolis, MN, USA), IL-21 (eBioscience, Vienna Austria), and TNF-alpha (R\&D Systems, Minneapolis, MN, USA). All analyses were performed following manufacturer's instructions.

\section{Flow cytometry}

Multi-color flow cytometry was performed on PBMCs. Fluorescence intensities were measured with a FACSCARIA (BD) and analyzed with FACSDiva version 6.1.3 (BD Bioscience, San Diego, CA, USA) using cutoffs based on isotype antibody staining. T-cell subpopulations were determined using the following fluorochrome-conjugated antibodies: PerCP-Cy5.5 anti-CD3, PE-Cy7 anti-CD4, FITC anti-CD8, in combination with Brilliant Violet 421 anti-CD38, V500 anti- HLA-DR (all purchased from BD Biosciences, San Diego, CA, USA).

B-cell subpopulations were determined using the following fluorochrome-conjugated antibodies: PE-Cy7 anti-CD19, FITC anti-CD10, PerCP-Cy5.5 anti-CD27, and APC anti-CD21 in combination with Brilliant Violet 421 anti-CD38, PE anti-IL21R (all purchased from BD Bioscience, San Diego, CA, USA). Dead cells were excluded using Fixable Viability Dye eFluor 780 obtained from eBioscience (San Diego, CA, USA).

\section{Statistical Methods}

Statistical analyses and graphical presentations were done using SPSS software, version 21.00 (IBM, Somers, NY, USA). Results are given as median, interquartile range (IQR), and percentage. Differences between groups were evaluated using the $\chi^{2}$ test or Fisher's exact test when appropriate for categorical variables and by KruskalWallis and Mann-Whitney $U$-test for quantitative variables. Post hoc analysis for pairwise comparison was 
Table 1 Patient demographic and clinical characteristics. Values are expressed as median and interquartile range or percentage

\begin{tabular}{|c|c|c|c|c|c|}
\hline & HIV/UC & HIV & UC & All & $P$-values \\
\hline No. of patients & 7 & 9 & 10 & 26 & \\
\hline Age (yrs) & $51(34.0-64.0)$ & $46.0(30.0-64.0)$ & $40.0(32.0-52.0)$ & $43.0(34.0-60.0)$ & 0.424 \\
\hline Sex (male, \%) & 71.4 & 77.8 & 50.0 & 69.0 & 0.538 \\
\hline Smoking n (\%) & $1 / 7(14.3)$ & 3/9 (33.3) & $1 / 10(10.0)$ & 5/26 (19.2) & \\
\hline $\mathrm{BMI}$ & $24.8(21.4-30.7)$ & $25(22.4-28.0)$ & $24(21.4-31.5)$ & $24.4(21.7-29.6)$ & 0.993 \\
\hline Hemoglobin (g/dl) & $13.7(12.5-14.7)$ & $14.1(12.9-15.3)$ & $14.0(13.1-16.2)$ & $13.9(12.7-15.3)$ & 0.483 \\
\hline WBC (cells/ml) & 7390 (6160-8650) & $6000(5050-8100)$ & $5840(4550-7105)$ & $6180(5170-7600)$ & 0.231 \\
\hline Lymphocytes (cells/ml) & $2300(2111-2750)$ & $2270(1625-2500)$ & 1693 (1310-2024) & 2127 (1595-2490) & 0.083 \\
\hline \multicolumn{6}{|l|}{ IDB characteristics } \\
\hline Duration of UC (yrs) & $4.0(3.0-7.0)$ & - & $6.5(2.0-10.3)$ & $5.0(2.5-11.7)$ & 0.883 \\
\hline Disease activity $n(\%)$ & $1 / 7(14.3)$ & - & $0 / 10(0.0)$ & $1 / 17(5.9)$ & \\
\hline Disease location & $\begin{array}{c}\text { Pan colon }=2 \\
\text { Recto-sigma }=5\end{array}$ & - & $\begin{array}{c}\text { Pan colon }=5 \\
\text { Recto-sigma }=5\end{array}$ & $\begin{array}{c}\text { Pan colon }=7 \\
\text { Recto-siama }=10\end{array}$ & \\
\hline IBD pharmacotherapy & $7 / 7$ mesalazine & - & $10 / 10$ mesalazine & & 1.000 \\
\hline \multicolumn{6}{|l|}{ HIV characteristics } \\
\hline Time from HIV diagnosis & $11.0(8.0-12.0)$ & $7(4.5-2)$ & - & $10(5.3-11.7)$ & 0.286 \\
\hline CDC classification (A/B/C) & $4 / 3 / 0$ & $7 / 2 / 0$ & - & $11 / 5 / 0$ & 0.377 \\
\hline Route of infection & $7 / 7$ sexual & 9/9 sexual & - & $16 / 16$ sexual & 1.000 \\
\hline CD4 cell count (/mm3) & $672(511-1025)$ & $671(513-785)$ & - & $671(520-804)$ & 0.491 \\
\hline ART therapy & $\begin{array}{l}2 \text { - TDF, FTC, EFV } \\
2 \text { - TDF, FTC, RAL } \\
1 \text { - 3TC, ABC, EFV } \\
1 \text { - TDF, FTC, ATV/r } \\
1-\text { TDF, FTC, DRV/r }\end{array}$ & 9 - TDF, FTC, EFV & - & & \\
\hline
\end{tabular}

performed applying the Dunn-Bonferroni adjustments for multiple testing to detect differences between groups. Spearman's correlation coefficient was used to evaluate correlations between quantitative variables. Differences were considered statistically significant when $p<0.05$.

\section{Results}

\section{Patient characteristics}

A total of 26 individuals were enrolled in this cross-sectional study. Seven patients were HIV positive and affected by histologically diagnosed UC (group HIV/ UC), 9 were HIV-infected (group HIV), and 10 were HIV uninfected with UC and (group UC). Demographic data and disease(s) characteristics are reported in Table 1. No differences were observed between groups regarding age, sex, hematological, and biochemical parameters. Regarding UC localization, 2 of 7 and 5 of 10 patients in groups HIV/UC and UC, respectively, had pancolon involvement. They were all treated with mesalazine (dose ranging between 2.4 and $3.2 \mathrm{~g} / \mathrm{die}$ ); one patient in group HIV/UC was receiving a course of corticosteroids because of a clinical relapse. Immunovirological profile of HIVinfected patients was similar; all had been virologically suppressed for at least 1 year, and median number of CD4 cell was $671 / \mathrm{mm}^{3}$. ART regimens are reported in Table 1 and are only apparently variable, since all were based on a 3 drugs combination, with the same backbone (TDF/ FTC) in the majority (6 of 7) of HIV/UC subjects and in all (9 of 9) of HIV control group.

\section{Plasma markers of MT, enterocyte damage, inflammation, and immune activation}

Patients in group HIV had significantly higher levels of soluble CD14 (sCD14) (median: $2166 \mathrm{ng} / \mathrm{ml}$, IQR: $1618-2523, p=0.007$, Figure 1 A) and fatty acid-binding proteins (I-FABP) (median $906.7 \mathrm{pg} / \mathrm{ml}$ IQR: 667.51373.5, $p=0.001$ Table 2, Figure 2). A direct, although not significant, correlation was found between elevated levels of I-FABP and SCD14 levels $(r=0.355, p=0.076$ Figure 1B) across the total population, both HIV infected and uninfected. The humoral immune response to LPS, measured by EndoCAb IgG, did not reveal significant differences between groups (Table 2). Similarly, high-sensitive C-reactive protein (hs-CRP), TNF-alpha, IL-6, and IL-21 plasma levels were low or below detection limits, without differences between groups.

\section{$T$ - and B-cell subsets}

As expected, HIV-infected patients (groups HIV/UC and HIV) had significantly lower percentages of $\mathrm{CD} 3+/$ $\mathrm{CD} 4+(p=0.002)$ and higher percentages of $\mathrm{CD} 3+/ \mathrm{CD} 8+$ $(p=0.001)$ cells with respect to group UC (Table 3$)$. No differences were seen in the three groups in the percentage of B cells (data not shown).

\section{Discussion}

The main finding of our study, conducted in a population of HIV+ subjects successfully treated with ART, is the presence of lower plasma levels of sCD14 and I-FABP 


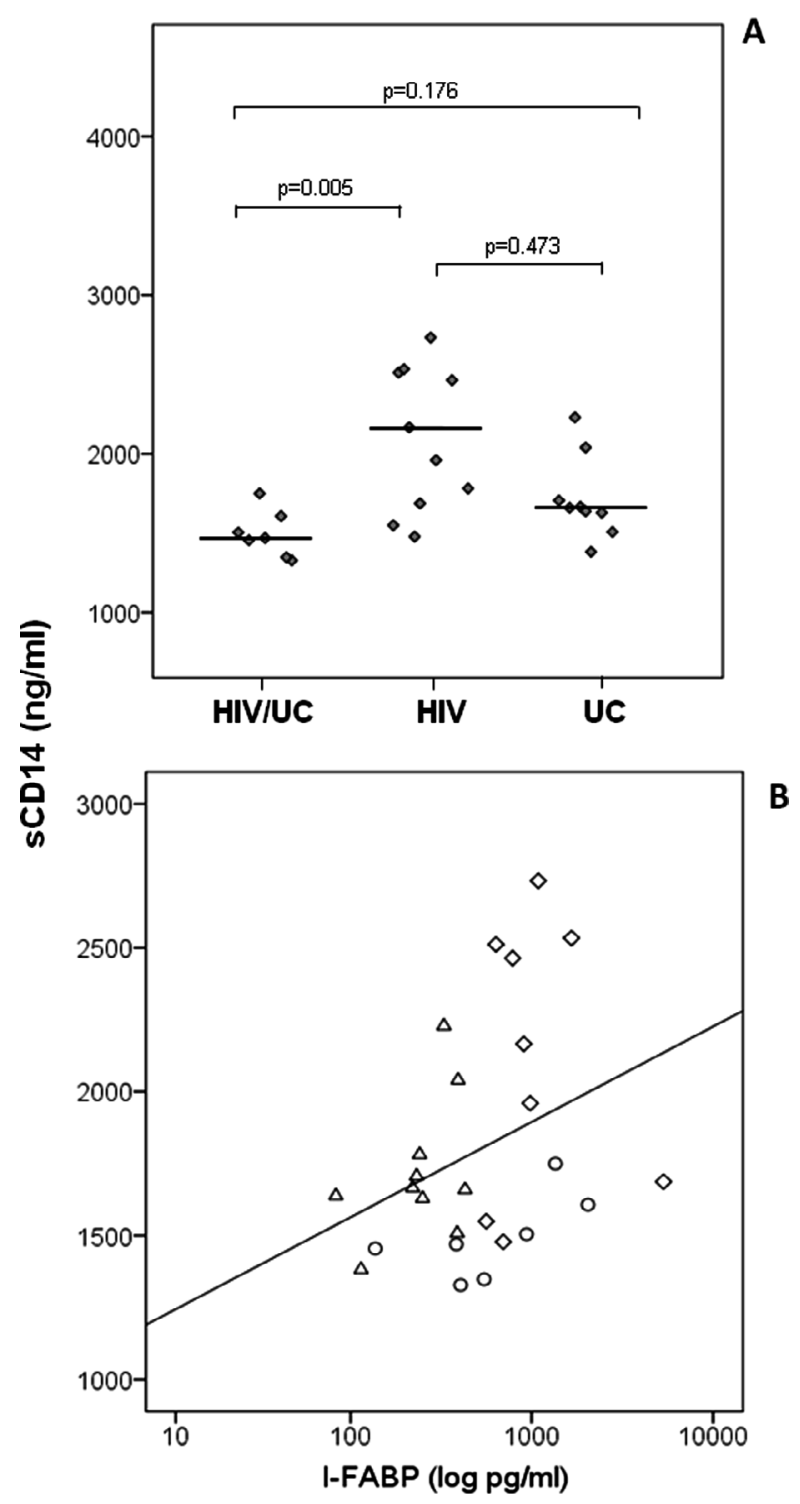

Figure 1 (A) Plasma levels of soluble CD14 (sCD14) in patients with concomitant HIV infection and UC (group HIV/ UC) with HIV infection (group HIV) and with UC (group UC). (B) Correlations between I-FABP and SCD14 plasma levels $(r=0.355, p=0.076)$. Groups are indicated with different dot shapes: group HIV/UC (circles); group HIV (diamonds); group UC: (triangles).

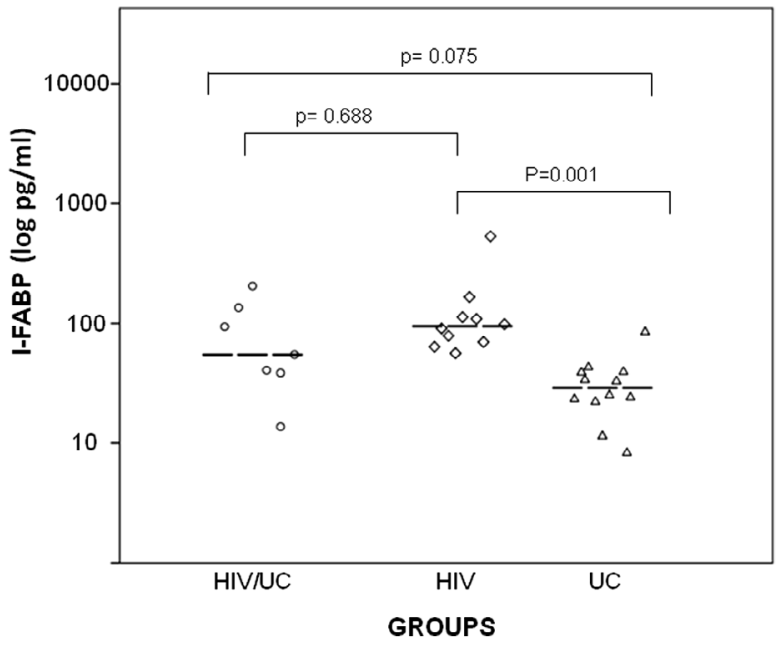

Figure 2 Plasma levels of intestinal fatty acid-binding protein (I-FABP) in patients with concomitant HIV infection and UC (group HIV/UC); with HIV infection (group HIV); with UC (group UC).

in those concomitantly affected by clinically stable UC. However, results and statistical analysis should be cautiously interpreted because of the small sample size due to the rarity of the study condition (UC in HIV-infected subjects). Under these premises, we may say that in our study, the presence of UC in HIV-infected subjects does not result in increased MT and gut mucosal damage.

Indeed, significantly higher plasma levels of sCD14 were found in group HIV compared both to HIV/UC and UC groups. Similarly, levels of I-FABP, a plasma marker of enterocyte damage, ${ }^{19}$ were higher in HIV patients respect to UC group. Recent studies have shown that I-FABP levels are associated with mortality and are inversely correlated with CD4 cell count in treated HIV infection, ${ }^{20}$ and elevated levels of this marker have been associated with the severity of disease in UC. ${ }^{21}$ In addition, a relationship has been reported between plasma levels of sCD14 and I-FABP and direct measures of gut epithelial damage and mucosal apoptosis, ${ }^{22,23}$ allowing us to suppose that also at the gut mucosal level, the concomitant

Table 2 Markers of MT, inflammation, and immune activation in plasma of patients. Values are expressed as median and interquartile range

\begin{tabular}{lcccc}
\hline Variables & HIV/UC & HIV & UC & $p$-values \\
\hline No. of patients & 7 & 9 & 10 & \\
I-FABP $(\mathrm{pg} / \mathrm{ml})$ & $547.6(384.9-1352.7)$ & $906.7(667.5-1373.5)$ & $245.8(194.1-390.2)$ & $0.001^{*}$ \\
EndoCAb $(\mathrm{GMU} / \mathrm{ml})$ & $85.6(61.4-107.2)$ & $100.4(40.9-173.6)$ & $75.8(51.8-96.9)$ & 0.852 \\
sCD14 $(\mathrm{ng} / \mathrm{ml})$ & $1469(1348-1607)$ & $2166(1618-2523)$ & $1663(1598-1846)$ & $0.007 \#$ \\
hsCRP $(\mathrm{mg} / \mathrm{ml})$ & $1.34(0.61-2.87)$ & $0.57(0.16-1.21)$ & $0.53(0.25-1.32)$ & 0.258 \\
IL-6 $(\mathrm{pg} / \mathrm{ml})$ & $1.59(1.06-6.24)$ & $1.90(0.62-3.87)$ & $1.22(0.36-1.40)$ & 0.211 \\
TNF-alpha $(\mathrm{pg} / \mathrm{ml})$ & bd & bd & bd & - \\
IL-21 $(\mathrm{pg} / \mathrm{ml})$ & $230.6(0.33-517.9)$ & $370.0(246.0-400.7)$ & $139.7(33.0-278.0)$ & 0.125 \\
\hline
\end{tabular}

Note: Results of pairwise comparison using the Dunn-Bonferroni post hoc approach were as follows:

*I-FABP: group HIV/UC vs. group HIV: $p=0.688$; group HIV/UC vs. group UC: $p=0.075$; group HIV vs. group UC: $p=0.001$. \#sCD14; group HIV/UC vs group HIV: $p=0.005$; group HIV/UC vs. group UC: $p=0.176$; group HIV vs group UC: $p=0.473$.

${ }^{\circ}$ Below detection limit (16.7 pg/ml). 
Table 3 Median and interquartile range of T-cell immunophenotyping percentage in the three groups

\begin{tabular}{|c|c|c|c|c|}
\hline & HIV/UC & HIV & UC & $p$-values \\
\hline No. of patients & 7 & 9 & 10 & \\
\hline CD3+/HLA-DR+ & $6.4(3.7-9.1)$ & $7.5(4.5-9.8)$ & $5.0(3.1-7.8)$ & 0.604 \\
\hline CD3+/CD4+ & $54.3(42.8-61.0)$ & $52.3(44.5-57.6)$ & $67.5(60.7-80.1)$ & $0.009^{*}$ \\
\hline CD3+/CD4+/CD38+ & $46.4(32.3-58.8)$ & $43.9(36.6-60.0)$ & $44.2(31.5-57.9)$ & 0.845 \\
\hline CD3+/CD4+/CD38+/HLADR & $4.6(2.3-6.7)$ & $3.2(2.6-7.4)$ & $3.9(2.6-6.7)$ & 0.998 \\
\hline CD3+/CD8+ & $37.6(35.0-51.2)$ & $42.4(34.2-48.7)$ & $25.5(10.0-29.5)$ & $0.003 \#$ \\
\hline CD3+/CD8+/CD38+ & $20.0(10.6-32.9)$ & $17.2(7.5-26.9)$ & $10.7(6.5-33.7)$ & 0.530 \\
\hline CD3+/CD8+/CD38+/HLADR & $14.8(10.9-15.8)$ & $19.1(6.4-33.2)$ & $19.8(11.8-34.0)$ & 0.434 \\
\hline
\end{tabular}

Note: Results of pairwise comparisons using the Dunn-Bonferroni post hoc approach were as follows:

${ }^{*} C D 3+/ C D 4+$; group HIV/UC vs. group HIV: $p=1.000$; group HIV/UC vs. group UC: $p=0.047$; group HIV vs. group UC: $p=0.015$. $\# C D 3+/ C D 8+$ : group HIV/UC vs. group HIV $p=1.000$; group HIV/UC vs. group UC: $p=0.030$; group HIV vs group UC: $p=0.011$.

presence of HIV infection and UC does not worsen and might even reduce the epithelial damage. It is worthy to emphasize that these observations only apply to UC, and in fact, a different pattern has been observed in subjects with Crohn's disease. ${ }^{24}$

It is not easy to explain our findings, although some evidence of an interplay between HIV infection and IBD were described in several case reports ${ }^{14-17}$ and in a more recent study by Viazis and colleagues, who suggested a protective role of HIV infection on the course of IBD, attributable to the HIV-related immunodeficiency. ${ }^{18}$ In our study, a factor that may have contributed to the observed results could be the long-term administration of mesalazine to patients with UC (groups HIV/UC and UC). This drug, a derivative of 5-aminosalicylic acid (5-ASA), is the standard medication for mild to moderately active $\mathrm{UC}^{25,26}$ and may have influenced MT in HIV+ patients by its anti-inflammatory effect at GI mucosal level. Under this respect, it is worth mentioning that mesalazine is released in the distal ileum, which is one of the main sites of HIV replication in both acute and chronic phase of disease, even in subjects with suppressed viral replication. The potential role of mesalazine in HIV infection has been already explored by Somsouk et al. ${ }^{26}$ who performed a double-blind, placebo-controlled randomized clinical trial where mesalazine was administered for 12 weeks to HIV patients with incomplete immunologic recovery. Although this study failed to demonstrate favorable effects of mesalazine on either systemic or colonic immune activation, this could be partially attributable to the relatively short duration of treatment and the characteristics of patient population, since the same authors found that incomplete immunologic recovery is associated with limited regenerative activity of gut epithelium in response to mucosal damage. ${ }^{22}$ This could have blunted any potential effect of mesalazine.

Our study has important limitations. First, the small size of the population, which may be responsible for low statistical power thereby weakening our conclusions. However, this is related to the rarity of the condition we studied: by reviewing more than 1300 case records of HIV-positive subjects referred to one of the largest HIV clinical sites in Rome, only $0.69 \%$ were found to be affected by IBD, and all of them had UC. Therefore, enlarging sample size would be a rather unfeasible objective. A further limitation is that our study is a cross-sectional one collecting data at a single time point.

In conclusion, our results show that in a small population of ART-treated HIV-infected subjects affected by UC and on long-term treatment with mesalazine, MT and systemic inflammation are not enhanced, but seem rather mitigated. These data, although scarcely generalizable, provide a rationale basis for new randomized clinical trials with mesalazine or other gut-specific anti-inflammatory drugs, to explore whether they can represent a valid therapeutic approach to control MT in HIV patients.

\section{Competing interest}

None to declare. None of the authors has a commercial or other association, financial interest, activity, relationship, or association that might pose a conflict of interest.

\section{Acknowledgments}

We thank Mrs. Stefania Donnini for secretarial help. We are grateful to Dr. PG Pupino Carbonelli for hematological analysis. We thank Patrizia Cocco and Ferdinando Costa for technical support and all the patients participating in this study.

\section{References}

1 Brenchley JM, Douek DC. HIV infection and the gastrointestinal immune system. Mucosal Immunol. 2008;1:23-30. Review.

2 Costiniuk CT, Angel JB. Human immunodeficiency virus and the gastrointestinal immune system: Does highly active antiretroviral therapy restore gut immunity? Mucosal Immol. 2012;5:596-604.

3 MacArthur RD, DuPont HL. Etiology and pharmacologic management of noninfectious diarrhea in HIV-infected individuals in the highly active antiretroviral therapy era. Clin Infect Dis. 2012:55:860-867.

4 Brenchley JM, Price DA, Schacker TW, Asher TE, Silvestri G, Rao S, et al. Microbial translocation is a cause of systemic immune activation in chronic HIV infection. Nat Med. 2006;12:1365-1371.

5 Sandler NG, Koh C, Roque A, Eccleston JL, Siegel RB, Demino $\mathrm{M}$, et al. Host response to translocated microbial products predicts 
outcomes of patients with HBV or HCV infection. Gastroenterology. 2011;141:1220-1230.

6 Pinzone MR, Celesia BM, Di Rosa M, Cacopardo B, Nunnari G. Microbial Translocation in Chronic Liver Diseases. Int J Microbiol. 2012; Article ID 694629, p. 12.

7 French AL, Evans CT, Agniel DM, Cohen MH, Peters M, Landay $\mathrm{AL}$, et al. Microbial translocation and liver disease progression in women coinfected with HIV and hepatitis C virus. J Infect Dis. 2013;208:679-689.

8 Giannelli V, Di Gregorio V, Iebba V, Giusto M, Schippa S, Merli $\mathrm{M}$, et al. Microbiota and the gut-liver axis: Bacterial translocation, inflammation and infection in cirrhosis. World $\mathrm{J}$ Gastroenterol. 2014;20:16795-16810.

9 Funderburg NT, Stubblefield Park SR, Sung HC, Hardy G, Clagett $\mathrm{B}$, Ignatz-Hoover J, et al. Circulating CD4(+) and CD8(+) T cells are activated in inflammatory bowel disease and are associated with plasma markers of inflammation. Immunology. 2013;140:87-97.

10 Sobczak M, Fabisiak A, Murawska N, Wesołowska E, Wierzbicka $\mathrm{P}$, Wlazłowski M, et al. Current overview of extrinsic and intrinsic factors in etiology and progression of inflammatory bowel diseases. Pharmacol Rep. 2014;66:766-775.

11 Kamat A, Ancuta P, Blumberg RS, Gabuzda D. Serological markers for inflammatory bowel disease in AIDS Patients with evidence of microbial translocation. PLoS ONE. 2010;5:e15533.

12 Soria A, Rossi M, Muscatello A, Gori A. HIV testing: A must for patients with inflammatory bowel disease? Am J Gastroenterol. 2011;106:1727-1728.

13 Landy J, Gazzard B, Harbord M. Inflammatory bowel disease in HIV seropositive individuals: Analysis of a large cohort. Gastroenterology. 2008; 134: A-504.

14 Lautenbach E, Lichtenstein GR. Human immunodeficiency virus infection and crohn's disease: The role of the CD4 cell in inflammatory bowel disease. J Clin Gastroenterol. 1997;25:456-459.

15 Pospai D, René E, Fiasse R, Farahat K, Beaugery L, Lammens P, et al. Chron's disease stable remission after human immunodeficiency virus infection. Dig Dis Sci. 1998;43:412-419.

16 Franke M, Kruis W, Heitz W. First manifestation of ulcerative colitis in a patient with HIV infection. Gastroenterology. 1990;98:544-545.
17 Bernstein CN, Snape WJ Jr. Active idiopathic ulcerative colitis in a patient with ongoing HIV-related immunodepression. Am J Gastroenterol. 1991;86:907-909.

18 Viazis N, Vlachogiannakos J, Georgiou O, Rodias M, Georgiadis $\mathrm{D}$, Papastamopoulos V, et al. Course of inflammatory bowel disease in patients infected with human immunodeficiency virus. Inflamm Bowel Dis. 2010;16:507-511.

19 Pelsers MM, Hermens WT, Glatz JF. Fatty acid-binding proteins as plasma markers of tissue injury. Clin Chim Acta. 2005;352:15-35. Review.

20 Hunt PW, Sinclair E, Rodriguez B, Shive C, Clagett B, Funderburg $\mathrm{N}$, et al. Gut epithelial barrier dysfunction and innate immune activation predict mortality in treated HIV infection. J Infect Dis. 2014;210:1228-1238.

21 Wiercinska-Drapalo A, Jaroszewicz J, Siwak E, Pogorzelska J, Prokopowicz D. Intestinal fatty acid binding protein (I-FABP) as a possible biomarker of ileitis in patients with ulcerative colitis. Regul Pept. 2008;147:25-28.

22 Somsouk M, Estes JD, Deleage C, Dunham RM, Albright R, Inadomi $\mathrm{JM}$, et al. Gut epithelial barrier and systemic inflammation during chronic HIV infection. AIDS. 2015;29:43-51.

23 Hoffmanová I, Sánchez D, Hábová V, Anděl M, Tučková L, Tlaskalová-Hogenová H. Serological markers of enterocyte damage and apoptosis in patients with celiac disease, autoimmune diabetes mellitus and diabetes mellitus type 2 [Epub ahead of print December 3, 2014]. Physiol Res. 2015;64:537-546.

24 Marchioni Beery R, Kane S. Current approaches to the management of new-onset ulcerative colitis. Clin Exp Gastroenterol. 2014;7:111132.

25 Criscuoli V, Modesto I, Orlando A, Cottone M. Mesalazine for the treatment of inflammatory bowel disease. Expert Opin Pharmacother. 2013;14:1669-1678.

26 Somsouk M, Dunham RM, Cohen M, Albright R, Abdel-Mohsen M, Liegler T, et al. The immunologic effects of mesalamine in treated HIV-infected individuals with incomplete CD4+ T cell recovery: A randomized crossover trial. PLoS One. 2014;9:e116. 